\title{
Prolonged Disease Stability With Trabectedin in a Heavily Pretreated Elderly Patient With Metastatic Leiomyosarcoma of the Thigh and Renal Failure: A Case Report and Review of the Literature
}

\author{
Danilo Galizia, Erica Palesandro, Anna Maria Nuzzo, Ymera Pignochino, \\ Sandra Aliberti, Massimo Aglietta, and Giovanni Grignani \\ Medical Oncology Unit, Institute for Cancer Research and Treatment, \\ Fondazione del Piemonte per l' Oncologia Candiolo, Turin, Italy
}

\begin{abstract}
Leiomyosarcoma represents about $24 \%$ of all soft tissue sarcomas and can originate from retroperitoneum, uterus, or extremities. Adequate local control may be achieved with surgery and radiotherapy. In the presence of unresectable metastases either doxorubicin- or gemcitabine-based chemotherapy are the standard of treatment. Nevertheless, prognosis remains poor regardless of the selected chemotherapy regimen, and new effective therapeutic agents for patients with advanced leiomyosarcoma are needed. Trabectedin, a promising new DNA-damaging agent with a mechanism of action that is different from that of traditional alkylating agents, is approved in Europe for the treatment of patients with advanced soft tissue sarcoma, after failure of anthracyclines and ifosfamide, or who are unsuited to receive these agents and in combination with pegylated liposomal doxorubicin (PLD) for the treatment of patients with relapsed platinum-sensitive ovarian cancer. We present a case of a 76-year-old patient with progressive metastatic lung lesions from a previously resected primary leiomyosarcoma of the thigh and moderate renal failure, who achieved 17 months of disease stability during third-line treatment with trabectedin. Trabectedin was not associated with any cumulative toxicity and was consistently well tolerated for a total of 22 treatment cycles. Current evidence on trabectedin is also presented.
\end{abstract}

Key words: Leiomyosarcoma; Lung metastases; Soft tissue sarcoma; Thigh; Trabectedin

\section{INTRODUCTION}

Leiomyosarcoma is one of the most frequent subtypes of soft tissue sarcoma, a rare group of pathologically heterogeneous tumors of mesenchymal origin (1). Leiomyosarcoma can occur anywhere in the body but is best known as a tumor occurring in the uterus, retroperitoneal space, and nonvisceral soft tissue $(2,3)$. Although adequate local control may be achieved with surgery and radiotherapy, the long-term prognosis for patients with nonvisceral soft tissue leiomyosarcoma is poor (3). The site of the primary tumor is an important prognostic factor, and differences in response to chemotherapy are seen for leiomyosarcomas from different anatomical sites, with uterine leiomyosarcoma being relatively responsive to chemotherapy, and extremity leiomyosarcomas demonstrating low-to-moderate chemosensitivity $(4,5)$.

Anthracycline-based chemotherapy has been standard treatment for soft tissue sarcoma since the mid-1970s (5). Although not statistically significant, in a review of over 2,000 patients involved in European Organization for
Research and Treatment of Cancer, Soft Tissue, and Bone Sarcoma Group studies, leiomyosarcomas appeared to have poorer response rates to anthracyclines than other histological subtypes of soft tissue sarcoma (6), but these studies were performed before it was realized that most gastrointestinal leiomyosarcomas are actually chemoresistant gastrointestinal stromal tumors (5). In general, responses to standard first-line anthracycline-based chemotherapy are short-lived. Single-agent gemcitabine has shown limited activity in soft tissue sarcoma in general, with the exception of leiomyosarcoma and liposarcoma (5). As a second-line strategy, gemcitabine with docetaxel may only achieve $32 \%$ of best overall response with a median progression-free survival of 6.2 months (7).

Earlier this year, the US FDA and European Medicines Agency approved the multitargeted angiogenesis inhibitor pazopanib for the treatment of advanced soft tissue sarcoma in patients who have received prior chemotherapy based on the results of the randomized, placebocontrolled, phase III PALETTE trial (8). However, the

Address correspondence to Danilo Galizia, M.D., Medical Oncology Unit, Institute for Cancer Research and Treatment, Fondazione del Piemonte per l' Oncologia, Strada Provinciale 142 km 3,95 10060 Candiolo, Torino, Italy. Tel: +390119933623; Fax: +390119933290; E-mail: danilo.galizia@ircc.it 
efficacy of this molecule for the treatment of patients with adipocytic STS or gastrointestinal stromal tumors (GIST) has not been demonstrated to date. Moreover, a number of novel agents are under investigation for the treatment of soft tissue sarcoma, including the microtubule inhibitor eribulin, agents targeting mammalian target of rapamycin, or insulin-like growth factor receptor. Other approaches, as multitargeted tyrosine kinase inhibitors, including sorafenib and sunitinib, failed to obtain satisfying results to date.

In 2007, trabectedin was approved in Europe for the treatment of patients with advanced soft tissue sarcoma after failure of anthracyclines and ifosfamide or who are unsuited to receive these agents.

Trabectedin, a tetrahydroisoquinoline alkaloid isolated from the Caribbean marine tunicate Ecteinascidia turbinata and now produced synthetically, has been shown active in soft tissue sarcomas $(5,9)$. Trabectedin has a unique mechanism of action that involves binding to the minor DNA groove and subsequent bending of DNA toward the major groove, thereby interfering with DNA repair and transcription processes, resulting in cell cycle perturbations and cell death (10).

We present a case of a patient with metastatic lung lesions from a primary leiomyosarcoma of the thigh and moderate renal failure who had prolonged stabilization of disease during third-line treatment with trabectedin. The feasibility of trabectedin treatment in patients with renal impairment has been poorly investigated. While a recent case report examined trabectedin pharmacokinetics in a patient on hemodialysis (11), to our knowledge the effectiveness and safety of trabectedin has not been investigated in patients with moderate renal failure. We also present a summary of current evidence on trabectedin.

\section{CASE REPORT}

In January 2002, a 69-year-old male was admitted to our hospital with a palpable mass in the right thigh. His medical history included type 2 diabetes mellitus treated with repaglinide, arterial hypertension treated with amlodipine, dyslipidemia treated with pravastatin, and benign prostatic hypertrophy treated with tamsulosin and finasteride. With the exception of blood glucose levels, blood count and biochemistry parameters were normal. Magnetic resonance imaging of the right thigh showed a lateral spindle cell mass that was $7 \mathrm{~cm}$ in diameter. Computed tomography (CT) scan of the chest showed no lung metastases. The right thigh mass was successfully resected with wide margins. The pathological report was consistent with a high-grade pleomorphic sarcoma and a histological diagnosis of leiomyosarcoma. Four cycles of intravenous adjuvant chemotherapy with epirubicin $\left(60 \mathrm{mg} / \mathrm{m}^{2}\right.$ on days 1 and 2 ) and ifosfamide ( $3 \mathrm{~g} / \mathrm{m}^{2}$ on days $\left.1-3\right)$ repeated every 21 days, and radiotherapy (66 Gy total) were administered.
Toxicity was as expected: grade 2 mucositis and grades 3-4 neutropenia, anemia, and thrombocytopenia.

The patient was well until February 2007, when a follow-up CT scan revealed a 6-mm node in the superior lobe of the left lung. Fluorodeoxyglucose-positron emission tomography (FDG-PET) scan confirmed the presence of an isolated lesion in the left lung with increased metabolic activity. In March 2007, resection of this node was performed, with histological confirmation of leiomyosarcoma. Six months later, three new lung nodules, the largest of which was $1.4 \mathrm{~cm}$ in diameter, were detected by a CT scan. The patient refused surgery and specified that he wished to avoid chemotherapy that would cause alopecia and mucositis. Gemcitabine $\left(1 \mathrm{~g} / \mathrm{m}^{2}\right)$ on days 1,8 , and 15 repeated every 3 weeks was started, and after two cycles, CT scan demonstrated stable disease. A total of eight cycles of gemcitabine were administered, with a best response of stable disease. The main gemcitabine-associated toxicities were grade 2 fatigue, grade 2 thrombocytopenia, and fever. After the last cycle of gemcitabine in October 2008, the patient asked for a rest period, and a new follow-up phase started.

In March 2009, CT scan showed numeric and dimensional progression of metastatic lung disease, according to RECIST criteria (12), with the largest nodule now $3 \mathrm{~cm}$ in diameter. In the meantime, patient's renal function gradually worsened, reaching a nadir creatinine clearance of $45 \mathrm{ml} / \mathrm{min}$, calculated with Cockroft-Gault formula. We believe this mild renal insufficiency development was due to hypertensive plus diabetic nephropathy. Even if $1 \mathrm{~L}$ of saline solution plus acetilcysteine were administered before and after each CT scan, surely a role in kidney function worsening was played by iodine contrast medium use.

Second line treatment with trabectedin $\left(1.3 \mathrm{mg} / \mathrm{m}^{2} /\right.$ $24 \mathrm{~h}$ infusion through a central venous line every 21 days) was begun, with dexamethasone $8 \mathrm{mg}$ administered the day before and tapered in 4 days after each administration of trabectedin. The patient received a total of 22 cycles of trabectedin without experiencing any toxicities other than grade 1 leucopenia, grade 1 increases in aspartate aminotransferase (AST) and alanine aminotransferase (ALT) levels, and grade 2 fatigue, so no dose adjustment was required. Renal function remained stable, and hypertension was well controlled. Echocardiography, which was performed every 6 months, did not reveal any clinically relevant abnormalities requiring dose modification. Our patient did not report any respiratory symptoms, such as cough or dyspnea. During trabectedin treatment, CT scans, which were performed every 90 days, recorded no progression over 17 months (Fig. 1). In December 2010, on the basis of the patient's own decision, treatment was interrupted. At the last follow-up visit, in July 2011, the patient was alive and felt well, and according to RECIST criteria, there was a dimensional progression on CT scan. 


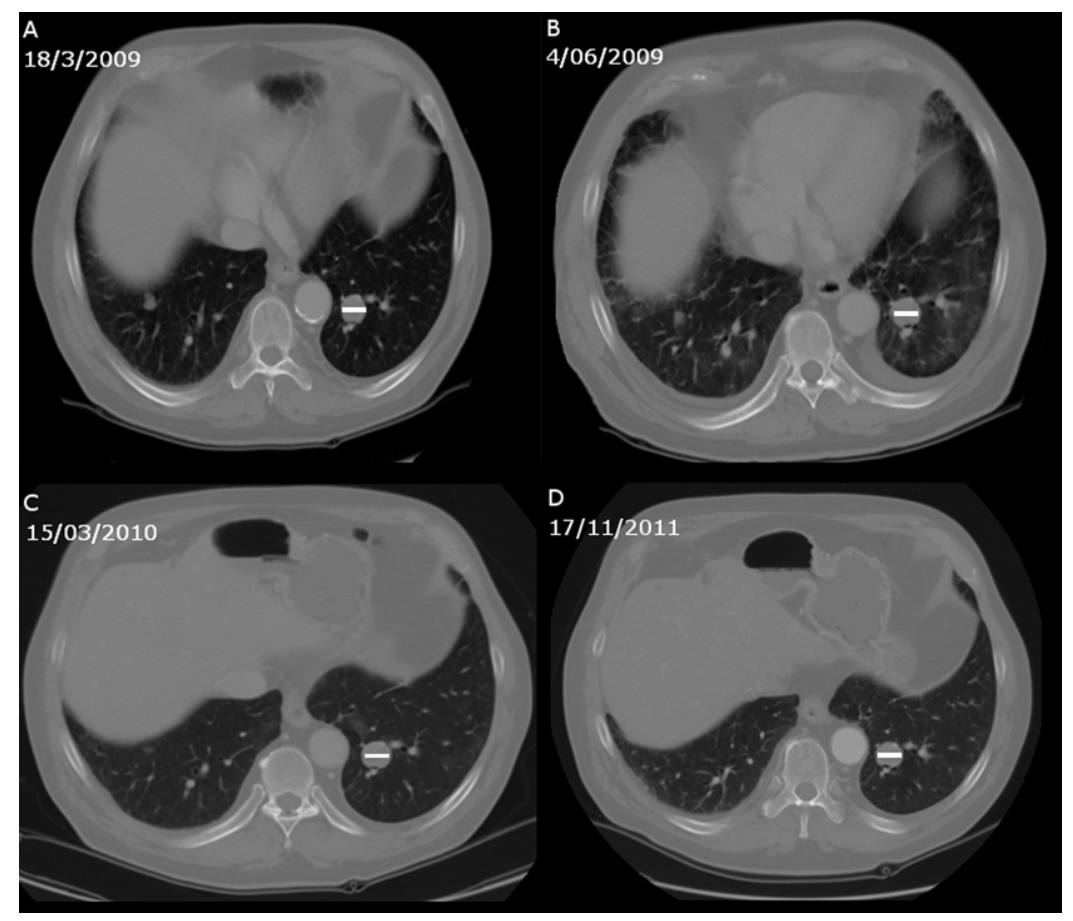

Figure 1. Chest CT scan before (A) and after 3 (B), 12 (C), and 22 (D) cycles of trabectedin. Diameter (white bar) is $3 \mathrm{~cm}$.

\section{TRABECTEDIN: CURRENT EVIDENCE}

\section{Pharmacology of Trabectedin}

Although the mechanism of action of trabectedin is not fully understood, it has been studied in several preclinical in vitro and in vivo models and according to recent evidence can be considered as a new multitarget chemotherapeutic agent. In contrast to traditional alkylating agents, which bind guanine at the N7 or O6 position in the DNA major groove, trabectedin binds to the $\mathrm{N} 2$ amino group of guanines in the minor DNA groove, causing several antitumor effects at the transcriptional level (10). Trabectedin causes DNA structural modifications that may interfere with the recognition of specific DNA consensus sequences by some transcription factors, with in vitro studies showing that it affects transcription of activated genes, and does not seem to affect constitutive transcription (10). It is well known that trabectedin affects the tumor microenvironment. Indeed, when administrated at noncytotoxic concentrations, trabectedin inhibited the production by myxoid liposarcoma cells of various cytokines and chemokines involved in tumor growth and progression (13). Modulation of cytokines and chemokines occurs at the transcriptional level, indicating that the drug is active not only in cancer cells but also in some normal cells, such as macrophages or endothelial cells, which produce factors that are relevant for tumor growth and progression (10). Trabectedin-induced DNA damage is recognized by nucleotide excision repair (NER) pathways, resulting in inhibition of DNA repair proteins and induction of cell death. Therefore, in contrast to what would be expected from a traditional DNA-damaging agent, sensitivity to trabectedin is strictly correlated with NER functionality $(10,14)$. When NER does not work properly, for example, in xeroderma pigmentosum group $\mathrm{G}$ gene (XPG) mutations and/or certain excision repair cross complementing group 1 gene (ERCC1) polymorphisms, trabectedin cytotoxicity is expected to be low. In addition, trabectedin induces DNA double strand breaks that are repaired by the homologous recombination repair (HRR) pathway $(10,14)$. When there is low expression or inactivating mutations of HRR machinery, such as the breast cancer susceptibility 1 gene (BRCA1), trabectedin cytotoxicity is expected to be high.

Consistent with laboratory models, a retrospective study has shown that a composite DNA repair profile was predictive of response in patients with advanced sarcoma treated with trabectedin, whereby low BRCA1 and high ERCC1 and/or XPG expression was correlated with higher probability of response (14). The molecular characterization of soft tissue sarcomas with respect to their DNA repair machinery may have a clinically important impact on the individualization of treatment strategies (14). For example, loss of BRCA1 expression is known to occur in a subset of patients with uterine leiomyosarcoma (15), so it is possible that these patients may be 
particularly responsive to trabectedin. There have been no prospective studies of trabectedin in a selected population of patients with uterine leiomyosarcoma, but a recent retrospective analysis of 66 patients with advanced uterine leiomyosarcoma confirmed that trabectedin was active in a subgroup of heavily pretreated patients (objective RECIST response rate of $16 \%$ and stable disease in $35 \%$ of patients) (16). In a recent phase II study of trabectedin, in 20 patients with previously untreated advanced uterine leiomyosarcoma, there was a $10 \%$ partial response rate, but more than $50 \%$ of patients remained progression free and without treatment-ending toxicity for more than 6 months (10 cycles) (17). It is hoped that, in the future, it will be possible to identify subgroups of patients with leiomyosarcoma who are likely to do well on trabectedin on the basis of biomarkers such as BRCA1. This result is currently being studied prospectively in either BRCA mutated breast tumors or hereditary ovarian tumors and in patients with BRCAness phenotype (MITO 15 study; NCT00326456).

\section{Clinical Studies of Trabectedin as Second-Line or Salvage Therapy}

The pivotal study of trabectedin is a large open-label phase II study as second-line or salvage therapy in 270 patients with advanced or metastatic soft-tissue sarcoma (18). They were included in the study if they had advanced or metastatic leiomyosarcoma or liposarcoma with disease progression after at least one cycle of anthracycline- and ifosfamide-based chemotherapy. The investigator-assessed histology was leiomyosarcoma in approximately two-thirds of patients and liposarcoma in the remaining third of patients. The most common primary site of disease was retroperitoneal (23\% of patients), uterus (22\%), lower extremity (21\%), and other abdomen and pelvic sites (18\%). Patients were randomized 1:1 to receive trabectedin in two dosage schedules: $1.5 \mathrm{mg} / \mathrm{m}^{2}$ administered as a 24-h infusion once every 3 weeks or $0.58 \mathrm{mg} / \mathrm{m}^{2}$ administered as a 3-h infusion weekly for 3 weeks out of every four (18). Trabectedin $1.5 \mathrm{mg} / \mathrm{m}^{2}$ every 3 weeks was associated with a significantly greater TTP compared with trabectedin $0.58 \mathrm{mg} / \mathrm{m}^{2}$ weekly (3.7 vs. 2.3 months; $p=0.03$ ) (Table 1) (18). The median TTP was also significantly greater with three-weekly trabectedin compared with weekly dosing when investigator-reported data were examined (4.2 vs. 2.5 months; $p=0.005)$. A greater proportion of patients receiving three-weekly trabectedin compared with those receiving weekly dosing were progression-free at $3(52 \%$ vs. $45 \%)$ and $6(36 \%$ vs. $28 \%)$ months (Table 1) (18). Moreover, the median OS was 13.9 and 11.8 months in the respective groups. Over three times as many patients receiving three-weekly trabectedin experienced an objective response than those receiving weekly dosing (5.6\% vs. 1.6\%) (18). Two pooled analyses of phase II studies have examined the effect of age on the efficacy of trabectedin as second-line treatment of sarcoma and in the treatment of translocation-related sarcoma $(19,20)$. Demetri et al. showed that the efficacy of trabectedin $1.5 \mathrm{mg} /$ $\mathrm{m}^{2}$ every 3 weeks was not affected by patient age in their pooled analysis of five studies (21). Older patients $(n=83$; median age $=65$ years, range $=60-81$ years) and patients aged $<60$ years $(n=267$; median age $=48$ years $)$ both experienced an ORR of $10 \%$, with stable disease achieved in $47 \%$ and $40 \%$ of patients, respectively. PFS at 6 months was seen in $36 \%$ of elderly patients and $30 \%$ of younger patients; survival rates in elderly patients and younger patients were similar at 1 year $(56 \%$ and $55 \%)$ and 2 years $(38 \%$ and $29 \%)(21)$.

Approximately a quarter of sarcomas involves a chromosomal translocation (20) and a pooled analysis of data from 82 patients in eight studies showed that trabectedin was effective in this patient group (22). A partial

Table 1. Efficacy of Intravenous Trabectedin in the Treatment of Advanced or Metastatic Liposarcoma or Leiomyosarcoma After Failure of Prior Anthracycline- and Ifosfamide-Based Chemotherapy in a Randomized, Open-Label Phase II Study (18)

\begin{tabular}{lcccc}
\hline & & & \multicolumn{2}{c}{ Statistical Analysis } \\
\cline { 3 - 5 } & Arm A & Arm B & & \\
\hline Trabectedin dose & $1.5 \mathrm{mg} / \mathrm{m}^{2} \mathrm{q} 3 \mathrm{w}$ & $0.58 \mathrm{mg} / \mathrm{m}^{2} \mathrm{q} 1 \mathrm{w}$ $3 / 4$ weeks & \\
Administration & $24-\mathrm{h}$ infusion & $3-\mathrm{h}$ infusion & \\
No. of patients & 136 & 134 & \\
Median no. of cycles & 5 & 2 & \\
Median TTP (independent review; & 3.7 & 2.3 & $0.734(0.554,0.974)$ & 0.032 \\
primary endpoint) (mo) & & & & \\
Median TTP (investigator review) (mo) & 4.2 & 2.5 & $0.668(0.506,0.883)$ & 0.0046 \\
Median PFS (mo) & 3.3 & 2.3 & $0.755(0.574,0.992)$ & 0.0418 \\
Median OS (mo) & 13.9 & 11.8 & $0.843(0.653,1.090)$ & 0.192 \\
ORR (\%) & 5.6 & 1.6 & & \\
\hline
\end{tabular}

CI, confidence interval; HR, hazard ratio; mo, months; ORR, objective response rate; OS, overall survival; PFS, progression-free survival; q $x \mathrm{w}$, every $x$ weeks; TTP, time to progression. 
response was seen in $10 \%$ of patients and stable disease in $49 \%$ of patients. The median PFS was 4.1 months, and the median OS was 17.4 months. Eighty-one patients included in this analysis had received previous chemotherapy for sarcoma, and one patient was chemotherapynaive (22). A phase III study of trabectedin compared with a doxorubicin-based chemotherapy regimen as firstline therapy in translocation-related sarcoma is ongoing (NCT00796120-clinicaltrials.gov).

Trabectedin was effective in the treatment of advanced, recurrent myxoid liposarcoma, as assessed by study investigators, according to the results of a retrospective analysis of a trabectedin compassionate use program (23). Trabectedin was administered at doses of 1.1$1.5 \mathrm{mg} / \mathrm{m}^{2}$ administered over 3 or $24 \mathrm{~h}$ every 3 weeks. Of the 51 patients who received trabectedin, two achieved a complete response and 24 achieved partial response, making an ORR of $51 \%$. At 6 months, $88 \%$ of patients were progression free, and the median PFS was 14.0 months (23).

In addition to the phase III first-line study described above, trabectedin is currently being compared with dacarbazine for the treatment of advanced liposarcoma or leiomyosarcoma previously treated with anthracycline and ifosfamide in an American registration phase III trial (SAR 3007; NCT01343277). This study, and a number of phase II studies, should further define the place of trabectedin in the treatment of soft-tissue sarcoma.

\section{Clinical Studies as Neoadjuvant Therapy}

Preliminary results from a small phase II study suggested that trabectedin might be effective as neoadjuvant treatment of locally advanced (stage III) or recurrent, nonmetastatic, measurable myxoid/round cell liposarcoma (9). Using RECIST criteria, 5 of 19 (26\%) patients achieved a partial response and 14 (74\%) achieved stable disease. Patients received trabectedin $1.5 \mathrm{mg} / \mathrm{m}^{2}$ administered over $24 \mathrm{~h}$ every 3 weeks for three to six cycles prior to surgery (9). Data were included from 29 patients of which 19 received a complete course of trabectedin, underwent surgery, and were evaluable for RECIST response. Patients had not previously received chemotherapy or radiotherapy except for adjuvant postoperative radiotherapy (9). On these bases, the Italian Sarcoma Group has decided to launch a new prospective controlled randomized trial in patients with high-risk soft tissue sarcomas, comparing standard full-dose anthracycline + ifosfamide versus histotype tailored chemotherapy in five definite histotypes. [The selected histotype and the respective tailored systemic therapy are as follows: leiomyosarcoma-gemcitabine + dacarbazine; myxoid-round cell liposarcoma (cellular component $>5 \%$ ) - trabectedin; synovial sarcoma-high dose continuous infusion ifosfamide; malignant peripheral nerve sheath tumor- ifosfamide + etoposide; undifferentiated pleomorphic sarcoma-gemcitabine + docetaxel.]

\section{Tolerability of Trabectedin}

Grade 3/4 hematological adverse events occurring in the phase II study in patients with advanced or metastatic liposarcoma or leiomyosarcoma were neutropenia, thrombocytopenia, and anemia, which occurred in $47 \%$, $11 \%$, and $8 \%$ of patients receiving trabectedin $1.5 \mathrm{mg} /$ $\mathrm{m}^{2}$ three-weekly and $14 \%, 6 \%$, and $9 \%$ of those receiving trabectedin $0.58 \mathrm{mg} / \mathrm{m}^{2}$ (Fig. 2) (18). Neutropenia and thrombocytopenia of grade 3 or 4 were generally transient, persisting for 5-7 days, and resolving quickly. The incidence of febrile neutropenia was very low, occurring in $<1 \%$ of patients in each treatment arm (18).

The most frequently occurring biochemical abnormalities in patients receiving trabectedin were elevations in liver function tests (Fig. 2) (18). Grade 3/4 increases in ALT and AST occurred in $47 \%$ and $32 \%$ of patients receiving three-weekly trabectedin and $9 \%$ and $3 \%$ of those receiving weekly dosing. ALT and AST remained elevated for a median of 5-7 days and subsequent serious hepatic complications, such as jaundice and hepatic failure occurred in $<1 \%$ of patients (18). Grade $3 / 4$ elevations in creatine phosphokinase occurred in $5 \%$ of patients receiving trabectedin $1.5 \mathrm{mg} / \mathrm{m}^{2}$ every 3 weeks and in $10 \%$ of those receiving weekly dosing.

Rhabdomyolysis is a very rare toxic event, with an overall incidence of $0.7 \%$ as shown in a recent large analysis $(10,841$ patients: 2,789 from clinical trials; 3,926 from compassionate use programs; and 4,126 treated in the marketplace) (24). No predictive factors for this event were disclosed. Despite its low frequency, a routing monitoring of the creatine kinase level remains advisable $(24,25)$. In order to reduce the potential for trabectedin-induced hepatotoxicity and myelosuppression, dexamethasone premedication is necessary, starting from the day before trabectedin administration (26). A recent analysis of trabectedin-related cardiac adverse events retrieved from clinical trials, pharmacovigilance databases and spontaneously reported case revealed a low incidence, consisting mainly of arrhythmias, indicating that trabectedin has a low cardiac risk profile. The incidence of all grades of cardiac adverse events in a total of 1,132 patients treated with trabectedin weekly, every 3 weeks or every 4 weeks, was $<1 \%$ (27).

Subjective treatment-related adverse events included gastrointestinal effects and fatigue (18). Grade 3/4 fatigue, abdominal pain, nausea, and vomiting occurred in $8 \%$, $5 \%, 5 \%$, and $5 \%$ of patients receiving trabectedin $1.5 \mathrm{mg} /$ $\mathrm{m}^{2}$ every 3 weeks and $7 \%, 5 \%, 2 \%$ and $2 \%$ of patients receiving weekly dosing. Other adverse events often seen with other chemotherapy agents, such as alopecia, mucositis, neurotoxicity, and skin/nail toxicities occurred very rarely (18).
1

2

3

4

5

6 7 

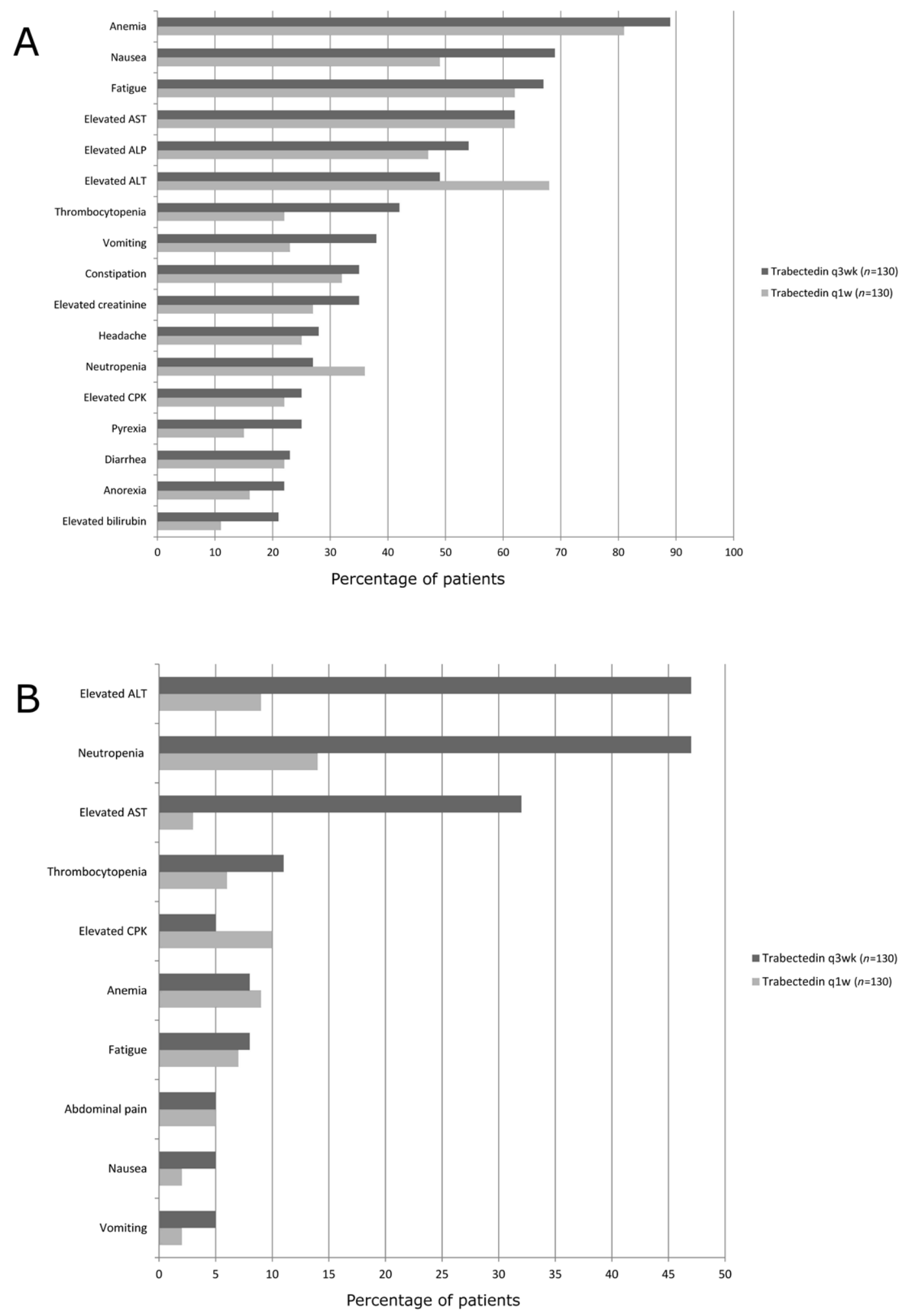

Figure 2. Incidence of (A) grade $1 / 2$ treatment-related adverse events occurring in $\geq 20 \%$ of patients and (B) grade $3 / 4$ treatmentrelated adverse events occurring in $\geq 5 \%$ of patients receiving intravenous trabectedin in a randomized, open-label phase II study (18). Patients had advanced or metastatic liposarcoma or leiomyosarcoma, had failed prior anthracycline- and ifosfamide-based chemotherapy, and received trabectedin $1.5 \mathrm{mg} / \mathrm{m}^{2}$ over $24 \mathrm{~h}$ every 3 weeks or $0.58 \mathrm{mg} / \mathrm{m}^{2}$ over $3 \mathrm{~h}$ weekly for 3 weeks of four. ALP, alkaline phosphatase; ALT, alanine aminotransferase; AST, aspartate aminotransferase; CPK, creatine phosphokinase; q $x \mathrm{w}$, every $x$ weeks. 


\section{DISCUSSION}

Just as there is some evidence suggesting that different histological subtypes of soft tissue sarcoma respond differently to chemotherapy, it is also reasonable to assume that leiomyosarcoma represents a spectrum of diseases with different sensitivities to chemotherapy (5). Leiomyosarcoma of nonvisceral soft tissue has not been well characterized; however, it is known that, in the presence of distant metastasis, either at the time of the diagnosis or afterwards, this disease is associated with a very poor prognosis, with a 5-year survival rate of $16 \%$ (3). Trabectedin has not been selectively studied in patients with leiomyosarcoma of non-visceral soft tissue. In the clinical trial by Demetri et al., the wide majority of treated leiomyosarcomas originated from uterus, retroperitoneum and other abdomen or pelvic sites (18). Our case specifically helps to clarify the utility of trabectedin in the treatment of patients with primary leiomyosarcoma of the nonvisceral soft tissue, in this case the thigh, which has metastasized and progressed after therapy with standard treatment options.

It is accepted practice that, in patients with leiomyosarcoma who maintain reasonable performance status, trabectedin may be standard option after the use of anthracycline based chemotherapy (4). Accordingly, our patient began second-line therapy with trabectedin after leiomyosarcoma of the thigh metastasized to the lung after surgery and adjuvant anthracycline-based chemotherapy and after progression of lung metastases, following therapy with gemcitabine. During 17 months of well tolerated treatment with trabectedin, our patient displayed consistently stable disease on chest CT scan, with no respiratory symptoms. As was the case with our patient, trabectedin is generally well tolerated in heavily pretreated patient populations, which, in conjunction with a lack of cumulative toxicity, allows patients who benefit from treatment to continue for a prolonged number of treatment cycles $(16,17)$. Nausea and vomiting are generally not serious with trabectedin, and fatigue is often the main subjective adverse event (25). Indeed, grade 2 fatigue was our patient's most severe complaint. Myelosuppression is the limiting toxicity of trabectedin (25), but despite grades 3 and 4 neutropenia and thrombocytopenia during anthracycline-based chemotherapy, our patient did not experience neutropenia or thrombocytopenia during therapy with trabectedin.

In conclusion, we reported the case of a heavily pretreated 76-year-old patient with metastatic leiomyosarcoma of the thigh and moderate renal failure, who achieved 17 months of disease stability during second-line trabectedin treatment. To our knowledge, this is the first report documenting an effective treatment with trabectedin in a patient with renal failure. Despite this concomitant condition and a number of important comorbidities, including type 2 diabetes and hypertension, our patient did not experience any major toxicity during 22 cycles of trabectedin therapy. For our patient with progressive metastatic lung disease, prolonged disease stabilization was a meaningful outcome, and trabectedin was instrumental in helping us to achieve our goals of care, which were to halt disease progression, while maintaining quality of life.

ACKNOWLEDGMENTS: Editorial assistance was provided by Luca Giacomelli, Ph.D., and Neil Reynolds, on behalf of in Science Communication, Springer Healthcare; this assistance was funded by Pharmamar. The authors declare no conflict of interest.

\section{REFERENCES}

1. Skubitz, K. M.; D'Adamo, D. R. Sarcoma. Mayo Clin. Proc. 8:1409-1432; 2007.

2. Fauth, C. T.; Bruecks, A. K.; Temple, W.; Arlette, J. P.; DiFrancesco, L. M. Superficial leiomyosarcoma: A clinicopathologic review and update. J. Cutan. Pathol. 37:269276; 2010.

3. Svarvar, C.; Böhling, T.; Berlin, O.; Gustafson, P.; Follerås, G.; Bjerkehagen, B.; Domanski, H. A.; Sundby Hall, K.; Tukiainen, E.; Blomqvist, C. Clinical course of nonvisceral soft tissue leiomyosarcoma in 225 patients from the Scandinavian Sarcoma Group. Cancer 109:282-291; 2007.

4. Collins, I. M.; Thomas, D. M. Novel approaches to treatment of leiomyosarcomas. Curr. Oncol. Rep. 13:316-322; 2011.

5. Scurr, M. Histology-driven chemotherapy in soft tissue sarcomas. Curr. Treat. Options Oncol. 12:32-45; 2011.

6. Van Glabbeke, M.; van Oosterom, A. T.; Oosterhuis, J. W.; Mouridsen, H.; Crowther, D.; Somers, R.; Verweij, J.; Santoro, A.; Buesa, J.; Tursz, T. Prognostic factors for the outcome of chemotherapy in advanced soft tissue sarcoma: An analysis of 2,185 patients treated with anthracyclinecontaining first-line regimens-a European Organization for Research and Treatment of Cancer Soft Tissue and Bone. J. Clin. Oncol. 17:150-157; 1999.

7. Maki, R. G.; Wathen, J. K.; Patel, S. R.; Priebat, D. A.; Okuno, S. H.; Samuels, B.; Fanucchi, M.; Harmon, D. C.; Schuetze, S. M.; Reinke, D.; Thall, P. F.; Benjamin, R. S.; Baker, L. H.; Hensley, M. L. Randomized phase II study of gemcitabine and docetaxel compared with gemcitabine alone in patients with metastatic soft tissue sarcomas: Results of Sarcoma Alliance for Research through Collaboration Study 002. J. Clin. Oncol. 25:2755-2763; 2007

8. van der Graaf, W. T.; Blay, J. Y.; Chawla, S. P.; Kim, D. W.; Bui-Nguyen, B.; Casali, P. G.; Schöffski, P.; Aglietta, M.; Staddon, A. P.; Beppu, Y.; Le Cesne, A.; Gelderblom, H.; Judson, I. R.; Araki, N.; Ouali, M.; Marreaud, S.; Hodge, R.; Dewji, M. R.; Coens, C.; Demetri, G. D.; Fletcher, C. D.; Dei Tos, A. P.; Hohenberger, P.; EORTC Soft Tissue and Bone Sarcoma Group; PALETTE study group. Pazopanib for metastatic soft-tissue sarcoma (PALETTE): A randomised, double-blind, placebo-controlled phase 3 trial. Lancet 19:1879-1886; 2012

9. Carter, N. J.; Keam, S. J. Trabectedin: A review of its use in soft tissue sarcoma and ovarian cancer. Drugs 70:355-376; 2010

10. D'Incalci, M.; Galmarini, C. M. A review of trabectedin (ET-743): A unique mechanism of action. Mol. Cancer Ther. 9:2157-2163; 2010 
11. Thariat, J.; Etienne-Grimaldi, M. C.; Launay-Vacher, V.; Soto-Matos, A.; Fernandez-Teruel, C.; Ghafari, T.; Marcy, P. Y.; Milano, G.; Renée, N.; Gastaud, L.; Thyss, A. Pharmacokinetics of trabectedin on hemodialysis: An application for the management of cancer patients with end-stage renal disease. Cancer Chemother. Pharmacol. 68:1363-1367; 2011.

12. Therasse, P.; Arbuck, S. G.; Eisenhauer, E. A.; Wanders, J.; Kaplan, R. S.; Rubinstein, L.; Verweij, J.; Van Glabbeke, M.; van Oosterom, A. T.; Christian, M. C.; Gwyther, S. G. New guidelines to evaluate the response to treatment in solid tumors. European Organization for Research and Treatment of Cancer, National Cancer Institute of the United States, National Cancer Institute of Canada. J. Natl. Cancer Inst. 92:205-216; 2000.

13. Germano, G.; Frapolli, R.; Simone, M.; Tavecchio, M. Erba, E.; Pesce, S.; Pasqualini, F.; Grosso, F.; Sanfilippo, R.; Casali, P. G.; Gronchi, A.; Virdis, E.; Tarantino, E.; Pilotti, S.; Greco, A.; Nebuloni, M.; Galmarini, C. M.; Tercero, J. C.; Mantovani, A.; D'Incalci, M.; Allavena, P. Antitumor and anti-inflammatory effects of trabectedin on human myxoid liposarcoma cells. Cancer Res. 70:2235-2244; 2010.

14. Schöffski, P.; Taron, M.; Jimeno, J.; Grosso, F.; Sanfilippo, R.; Casali, P. G.; Le Cesne, A.; Jones, R. L.; Blay, J. Y.; Poveda, A.; Maki, R. G.; Nieto, A.; Tercero, J. C.; Rosell, R. Predictive impact of DNA repair functionality on clinical outcome of advanced sarcoma patients treated with trabectedin: A retrospective multicentric study. Eur. J. Cancer 47:1006-1012; 2011.

15. Xing, D.; Scangas, G.; Nitta, M.; He, L.; Xu, X.; Ioffe, Y. J. M.; Aspuria, P. J.; Hedvat, C. Y.; Anderson, M. L.; Oliva, E.; Karlan, B. Y.; Mohapatra, G.; Orsulic, S. A role for BRCA1 in uterine leiomyosarcoma. Cancer Res. 69:8231$8235 ; 2009$.

16. Sanfilippo, R.; Grosso, F.; Jones, R. L.; Banerjee, S.; Pilotti, S.; D'Incalci, M.; Dei Tos, A. P.; Raspagliesi, F.; Judson, I.; Casali, P. G. Trabectedin in advanced uterine leiomyosarcomas: A retrospective case series analysis from two reference centers. Gynecol. Oncol. 123:553-556; 2011.

17. Monk, B. J.; Blessing, J. A.; Street, D. G.; Muller, C. Y.; Burke, J. J.; Hensley, M. L. A phase II evaluation of trabectedin in the treatment of advanced, persistent, or recurrent uterine leiomyosarcoma: A gynecologic oncology group study. Gynecol. Oncol. 124:48-52; 2012.

18. Demetri, G. D.; Chawla, S. P.; von Mehren, M.; Ritch, P.; Baker, L. H.; Blay, J. Y.; Hande, K. R.; Keohan, M. L.; Samuels, B. L.; Schuetze, S.; Lebedinsky, C.; Elsayed, Y. A.; Izquierdo, M. A.; Gómez, J.; Park, Y. C.; Le Cesne, A.
Efficacy and safety of trabectedin in patients with advanced or metastatic liposarcoma or leiomyosarcoma after failure of prior anthracyclines and ifosfamide: Results of a randomized phase II study of two different schedules. J. Clin. Oncol. 27:4188-4196; 2009.

19. Chawla, S.; Blay, J. Y.; Schuetze, S. Efficacy of secondline trabectedin in patients with advanced liposarcomas and leiomyosarcomas progressing despit prior conventional chemotherapy [abstract 9427]. Eur. J. Cancer 7(Suppl. 2): 598; 2009.

20. Le Cesne, A.; Gianni, L.; Maki, R. Translocation-related sarcomas (TRS): A retrospective analysis of activity with trabectedin [abstract 9401]. Eur J. Cancer 7(Suppl. 2):590; 2009.

21. Demetri, G.; Blay, J. Y.; Yovine, A. Efficacy and safety of trabectedin in soft tissue sarcoma (STS) are indpendent of patient age [abstract 9402]. Eur. J. Cancer 7(Suppl. 2):590; 2009.

22. Mertens, F.; Antonescu, C. R.; Hohenberger, P.; Ladanyi, M.; Modena, P.; D'Incalci, M.; Casali, P. G.; Aglietta, M.; Alvegård, T. Translocation-related sarcomas. Semin. Oncol. 36:312-323; 2009

23. Grosso, F.; Jones, R. L.; Demetri, G. D.; Judson, I. R.; Blay, J. Y.; Le Cesne, A.; Sanfilippo, R.; Casieri, P.; Collini, P.; Dileo, P.; Spreafico, C.; Stacchiotti, S.; Tamborini, E.; Tercero, J. C.; Jimeno, J.; D’Incalci, M.; Gronchi, A.; Fletcher, J. A.; Pilotti, S.; Casali, P. G. Efficacy of trabectedin (ecteinascidin-743) in advanced pretreated myxoid liposarcomas: A retrospective study. Lancet Oncol. 8:595-602; 2007.

24. Grosso, F.; D’Incalci, M.; Cartoafa, M.; Nieto, A.; FernándezTeruel, C.; Alfaro, V.; Lardelli, P.; Roy, E.; Gómez, J.; Kahatt, C.; Soto-Matos, A.; Judson, I. A comprehensive safety analysis confirms rhabdomyolysis as an uncommon adverse reaction in patients treated with trabectedin. Cancer Chemother. Pharmacol. 69:1557-1565; 2012

25. Casali, P. G.; Sanfilippo, R.; D’Incalci, M. Trabectedin therapy for sarcomas. Curr. Opin. Oncol. 22:342-346; 2010.

26. Grosso, F.; Dileo, P.; Sanfilippo, R.; Stacchiotti, S.; Bertulli, R.; Piovesan, C.; Jimeno, J.; D’Incalci, M.; Gescher, A.; Casali, P. G. Steroid premedication markedly reduces liver and bone marrow toxicity of trabectedin in advanced sarcoma. Eur. J. Cancer 42:1484-1490; 2006.

27. Lebedinsky, C.; Gómez, J.; Park, Y. C.; Nieto, A.; SotoMatos, A.; Parekh, T.; Alfaro, V.; Roy, E.; Lardelli, P.; Kahatt, C. Trabectedin has a low cardiac risk profile: A comprehensive cardiac safety analysis. Cancer Chemother. Pharmacol. 68:1223-1231; 2011. 